\title{
TROIS VESTIGES D'AQUEDUCS RURAUX GALLO-ROMAINS DANS LA PARTIE OCCIDENTALE DE LA CITÉ DE CARCASSONNE (AUDE)
}

\author{
Michel PASSELAC
}

Mots-clés. Aqueduc, eau courante, espace rural, irrigation, jardins d'agrément, technique de construction, villa.

Résumé. Les vestiges de trois conduites souterraines d'adduction d'eau ont été observés à la suite de prospections au sol et de travaux en milieu rural. Bien que de dimensions très modestes, ces aqueducs sont construits avec beaucoup de soin. Celui de Sur Fontorbe à Lavalette, près de Carcassonne, le mieux conservé, témoigne bien de l'attention apportée à ces ouvrages qui présentent la même structure que les grandes conduites. Dans deux cas, ces canalisations aboutissaient à une villa ; dans le troisième, il est également possible que l'aqueduc: alimentait un domaine. Les captages n'ont pas pu être localisés avec précision, mais on peut penser qu'ils prélevaient l'eau de petits ruisseaux alimentés par une source abondante ou un bassin versant. L'étude des sites et de leur environnement montre que l'eau n'y était pas rare. Des ruisseaux, des nappes phréatiques et une bonne pluviométrie constituaient des ressources suffisantes. La fonction de ces

conduites, dont le débit a été calculé, est ici discutée. Elles constituaient en effet pour le propriétaire du domaine un investissement non négligeable. Celui-ci était certainement justifié par des usages multiples, notamment ceux pour lesquels une eau abondante et courante. était particuliẹrement prisée : irrigation des cultures vivrières et des vergers à la saison sèche, arrosage et animation des jardins d'agrément. À partir de ces trois exemples, l'existence de dispositifs similaires peut être envisagée sur un certain nombre de villae de la région.

Key-words. Aqueduct, running water, rural space, irrigation, pleasure gardens, building techniques, villa.

Abstract. Remains of three underground channels have been detected during surve;, agricultural labour and public works in rural context. Despite small measurements, these aqueducts were carefully built. The best preserved, at Sur Fontorbe at Lavalette, near Carcassonne, shows the care given to these structures built the same way as vast conduits. Two of them run towards a villa, the third one could also carry water to an estate. Harnessings are not precisely located but it seems that water came from streams supplied by an abundant spring or a drainage basin. The study of the sites and their environment shows that water was not lacking. Streams and phreatic water were sufficient resources and local weather had a good pluviometry level. The flow of these channels has been calculated and their function is being discussed. For the landowner it was a valuable investment, justified by many different uses, in particular those which needed an abundant running water : irrigation for food crop and orchads during dry season, watering, pleasure gardens. From these three cases, similar arrangements are presumed on a series of villae in the area.

Translation : Isabelle FACDLFT

Schlagwörter. Römische Wasserleitung, fließendes Wasser, ländlicher Raum, Feldbewässerung, Ziergärten, Bautechniken, Villa.

Zusammenfassung. Im Zusammenhang mit Prospektionen und landwirtschaftlichen Arbeiten wurden Überreste von drei unterirdischen Wasserleitungen beobachtet. Sie haben zular nur bescheidene Dimension, sind aber mit großer Sorgfalt angelegt. Die am besten erhaltene, die von Sur Fontorbe bei Carcassonne, belegt sehr gut, wieviel Gewissenhaftigkeit man auf diese Bauwerke verwendet hat, die nach den gleichen Prinzipien errichtet sind wie die großen Anlagen. In zwei Fällen handelt es sich um Leitungskanäle, die zu einer Villa führten; bei dem dritten Beispiel wäre es sogar möglich, daß die Wasserleitung der Versorgung eines Domanialbetriebes diente. Die Fassungsbereiche konnten zuar nicht genau lokalisiest werden, aber es ist anzunehmen, daß die Leitungen jeweils kleinere, durch Quellen oder Quellhorizonte gespeiste Bachläufe zusammenfassen. Wie die Lntersuchung der Fundstellen und ihrer Lmgebung zeigt, war an Wasser jedenfalls kein Mangel. Oberflächengewüsser und Grundwasserstand hielten genügend Ressourcen bereit und das Klima der (iegend bietet ausreichende Niederschlagsmengen. Im vorliegenden Beitrag wird auch die Leistung der Wasserleitungen ermittelt und ihre Funklion diskutiert. Tatsächlich bedeutete ihre Einrirhtung für den Cutsbesitzer eine beträchtliche Investition. Iiese war aber sicherlich durch 
mehrfachen Nutzen gerechtfertigt. Vor allem ist hier an die besonderen Zwecke zu denken, die fließendes Wasser in großer Menge. erforderten, wie die Bewü̈sserung von Feldern, Nulzgärten und Obstkulturen während der Trockenperiode sowwie das Wässern und Betreiben von Ziergärten. Es ist zu vermuten, daß in der Region bei einer ganzen Anzahl von Villen Vorrichtungen in der Art der hier beschriebenen vorhanden waren.

Überselzung : Stefan WIRTH

Cet article a pour but de faire connaitre trois exemples de conduites d'eau maçonnées et enterrées découvertes en milieu rural, en relation avec des établissements gallo-romains (fig. 97). L'intérêt qui leur a été porté s'inscrit dans une démarche de constitution de données sur l'espace rural et l'agriculture antique dans l'ouest du bassin de l'Aude. Commencées dans les années 1980 (Passelac, 1983), ces recherches se sont appuyées sur des prospections au sol, des prospections aériennes, des photo-interprétations, des fouilles préventives, le suivi de travaux agricoles et la surveillance de travaux d'aménagement et de mise en place de réseaux récemment développée par la systématisation des diagnostics d'archéologie préventive.

Les trois conduites d'eau se trouvent dans la partie occidentale du territoire de la cité de Carcassonne; une région située à la limite des influences océaniques et méditerranéennes. Elle ne présente pas un climat particulièrement sec, comme celles, plus méditerranéennes ou plus méridionales, qui donnent généralement lieu à l'étude de ce genre d'ouvrage. Ces aqueducs constituaient-ils le seul moyen d'approvisionnement en eau des établissements ruraux pour lesquels ils ont été construits? Quelle quantité d'eau permettaient-ils d'acheminer, et peut-on en déduire l'usage qui en était fait ?

Nous décrirons d'abord les conduites, vestiges modestes mais témoins d'un soin particulier apporté à la recherche et à la maîtrise d'une eau abondante et courante, selon des techniques nouvelles. Comme il s'agit chaque fois d'observations très ponctuelles, nous chercherons ensuite à déterminer l'emplacement des captages, à approcher la pente et le débit des ouvrages, la quantité d'eau transportée et, à partir de

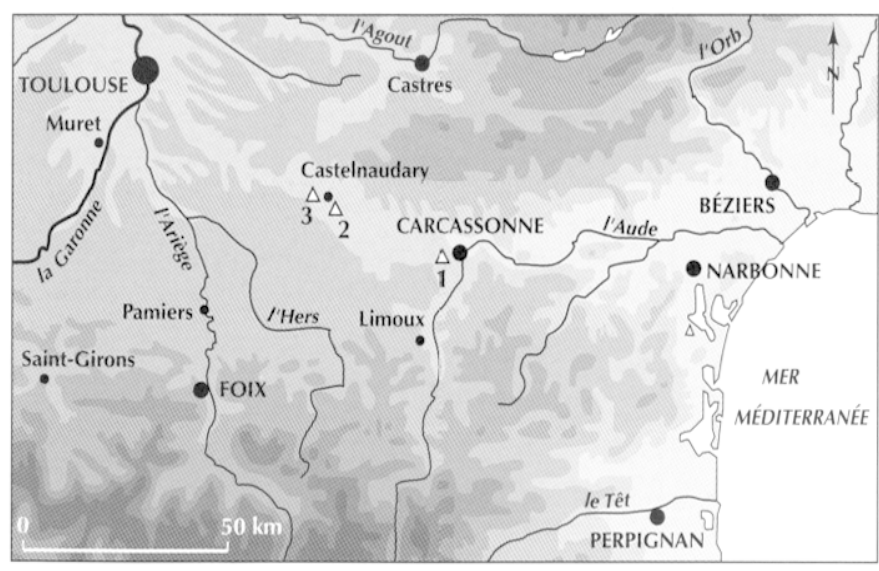

Fig. 97 - Localisation des sites étudiés dans le bassin occidental de l'Aude: 1, Sur Fontorbe à Lavalette et Carrassonne ; 2, Bartissol à Castelnaudary ; 3, La Baronne à Mas-Saintes-Puelles (DAO M. Passelac, CNRS). celle-ci, à formuler quelques hypothèses quant à l'utilisation de cette eau.

Dans cette région, aucune fouille extensive de villa n'a été réalisée. On dispose seulement d'explorations partielles d'établissements ruraux sur le terrain (Rancoule, 1988 ; Passelac, 1993), et de plans complets livrés par des prospections aériennes. Mais sur les clichés les conduites apparaissent rarement, et il est difficile de dire, sans indications sur la pente, s'il s'agit d'amenées ou d'évacuations. Aussi, on peut se demander si ces trois exemples sont des cas isolés ou si, au contraire, ils ne témoigneraient pas d'une pratique en définitive assez courante, mais restée encore méconnue, en matière d'équipement hydraulique dans nos régions relativement bien arrosées.

\section{DONNÉES DU TERRAIN}

\section{L'AQUEDUC DE SUR FONTORBE, COMMUNES DE LAVALETTE ET DE CARCASSONNE (AUDE)}

Cette conduite a été découverte en 1996 lors d'un défonçage pour la plantation d'une vigne. Intrigué par la distribution linéaire de matériaux de construction mis au jour dans un terrain limoneux, le propriétaire du terrain a sondé sa parcelle, repéré la conduite et procédé à son décapage mécanique sur une quarantaine de mètres environ. Informés de la découverte, nous avons pu en parfaire le décapage, l'observer et en effectuer le relevé ${ }^{75}$.

\section{LOCALISATION DU SEGMENT DE CONDUITE}

Ce segment d'aqueduc a été mis au jour à la limite des communes de Carcassonne et de Lavalette, à $400 \mathrm{~m}$ au nordnord-ouest du domaine de Fontorbe ${ }^{76}$. Le terrain forme ici une légère dépression d'orientation ouest-est, creusée entre deux croupes de grès et reliée au bassin du ruisseau de la Malpère tributaire de la proche rivière Aude. Le fond de cette dépression est parcouru par un fossé, ancien ruisseau coulant vers l'est (fig. 98). La conduite court du sud-est vers le nordouest, presque parallèle aux courbes de niveau, avec une faible

75. A. Bennes et G. Rancoule de la Société d'études scientifiques de l'Aude nous ont signalé les travaux du propriétaire, .M. P. Ormières. Nous avons distrait quelques fouilleurs du chantier alors en cours à Bram, ruc Marceau, pour réaliser cette opération. Celle-ci n’aurait pu être réaliscé sans ces précieuses collaborations et l'autorisation du Service régional de l'archéologie.

76. Laqueduc traversait dans sa partie médiane la parcelle 25 du cadastre de (arcassonne, section HI., et l'angle nord-est de la parcelle 18 du carlastre de Iaraleue, section AH (amnéc 1996). (arte topographique de l'IC:N au 1/25000, 2346 ouest, Limoux. 


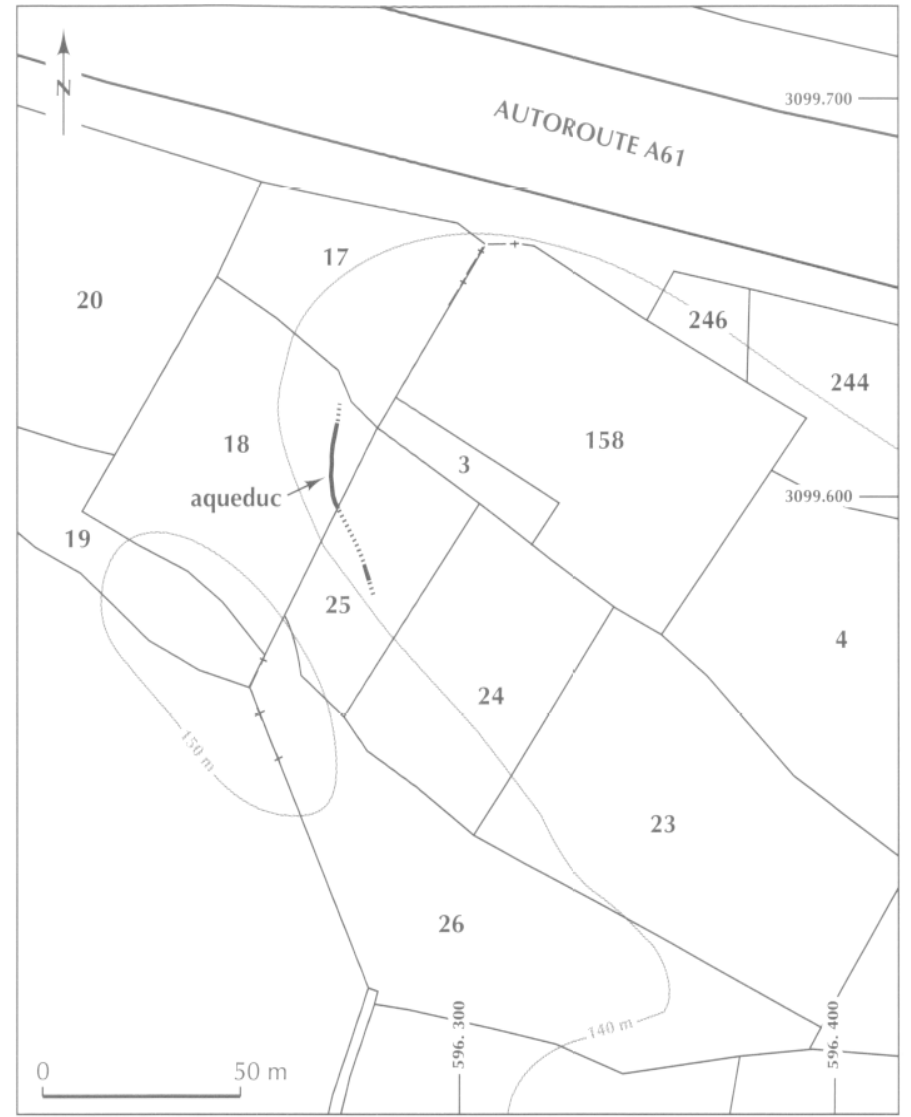

Fig. 98 - Localisation topographique et cadastrale de l'aqueduc de Sur Fontorbe à Lavalette et Carcassonne (DAO M. Passelac, CNRS).

pente. Bien conservée dans la partie basse de la parcelle, où elle a été protégée par les atterrissements, elle est très dégradée dans la zone haute. Dans cette dernière partie, les labours l'ont arasée jusqu'à son radier, puis, plus au sud encore, l'ont fait disparaître. Il est donc impossible d'en prolonger le tracé vers le sud, alors que de nouvelles explorations pourraient la retrouver vers le nord.

\section{DESCRIPTION DE LA CONDUITE}

La conduite a été reconnue par une tranchée large de $2 \mathrm{~m}$ et longue de $20 \mathrm{~m}$, puis, après une interruption de $15 \mathrm{~m}$, par une nouvelle tranchée de $4 \mathrm{~m}$ de long (fig. 99 et 100). Au total le segment relevé mesure donc $39 \mathrm{~m}$. Il présente dans sa partie centrale un infléchissement vers le nord qui lui donne un tracé sensiblement parallèle à la courbe de niveau des $140 \mathrm{~m}$.

Dans les parties médiane et basse de la parcelle, la conduite a été construite dans une tranchée large de $0,80 \mathrm{~m}$, creusée dans des limons bruns. Le niveau du sol antique à partir duquel elle a été mise en place n'a pu être déterminé, car bouleversé par les labours. Aussi ne peut-on déterminer exactement la profondeur de l'enfouissement de l'ouvrage dans l'Antiquité. La couverture de l'aqueduc se trouvait sous $0,85 \mathrm{~m}$ de limons dans le bas de la parcelle, de $0,75 \mathrm{~m}$ dans sa partie médiane. Au sud, le niveau d'arasement était visible à $0,60 \mathrm{~m}$ sous le sol actuel.

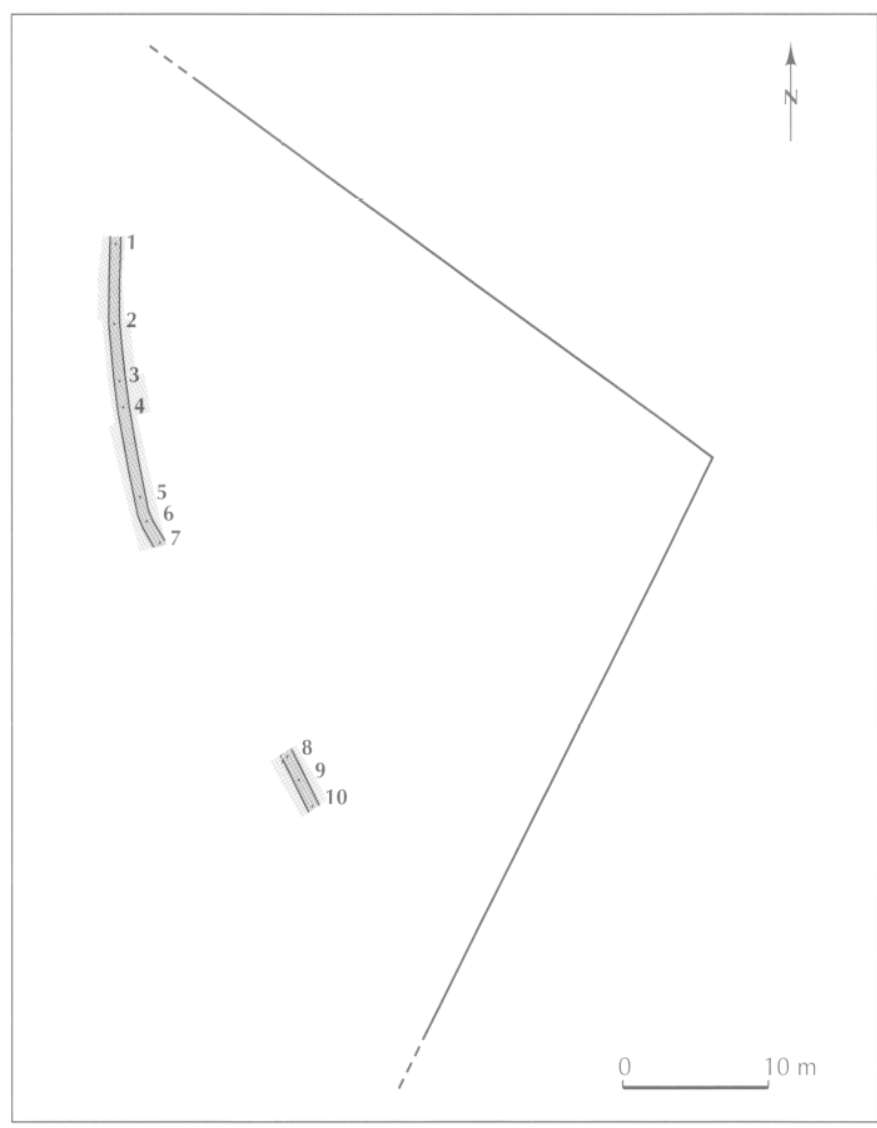

Fig. 99 - Sur Fontorbe à Lavalette et Carcassonne : plan du chantier et localisation des points d'observation et de mesures (DAO M. Passelac, CNRS).

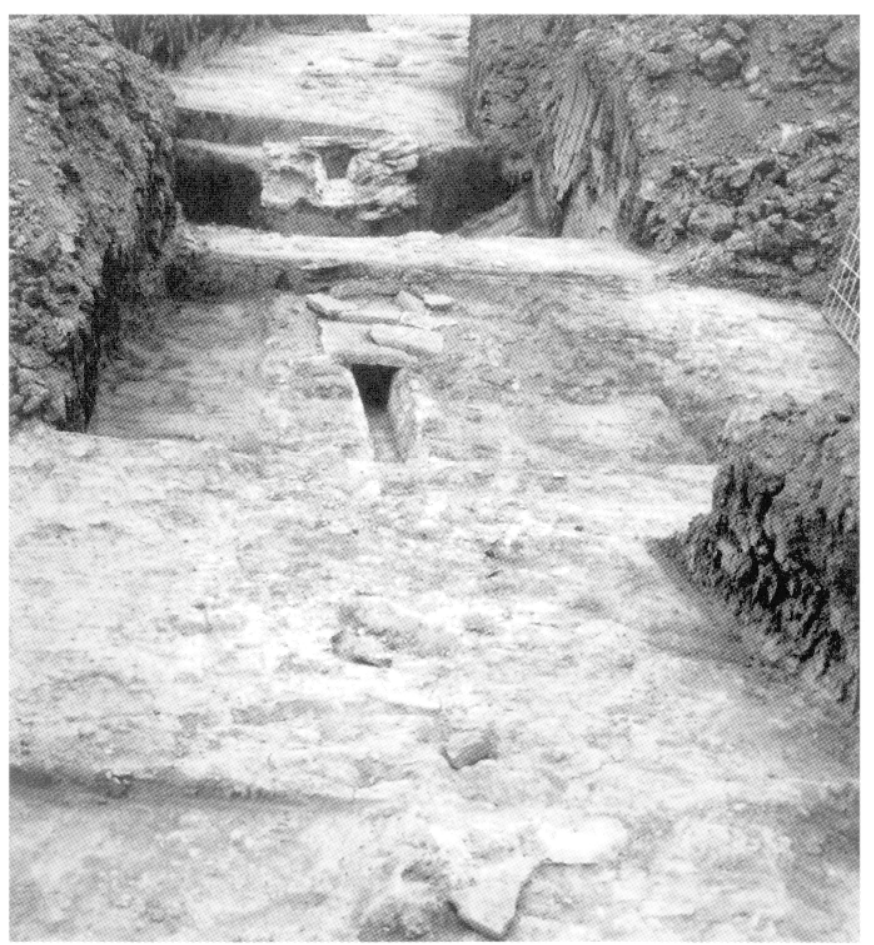

Fig. 100 - Sur Fontorbe à Lavalette et Carcassonne : l'aqueduc vu du sud, dans la partie centrale du chantier (cliché M. Passelac, CNRS). 

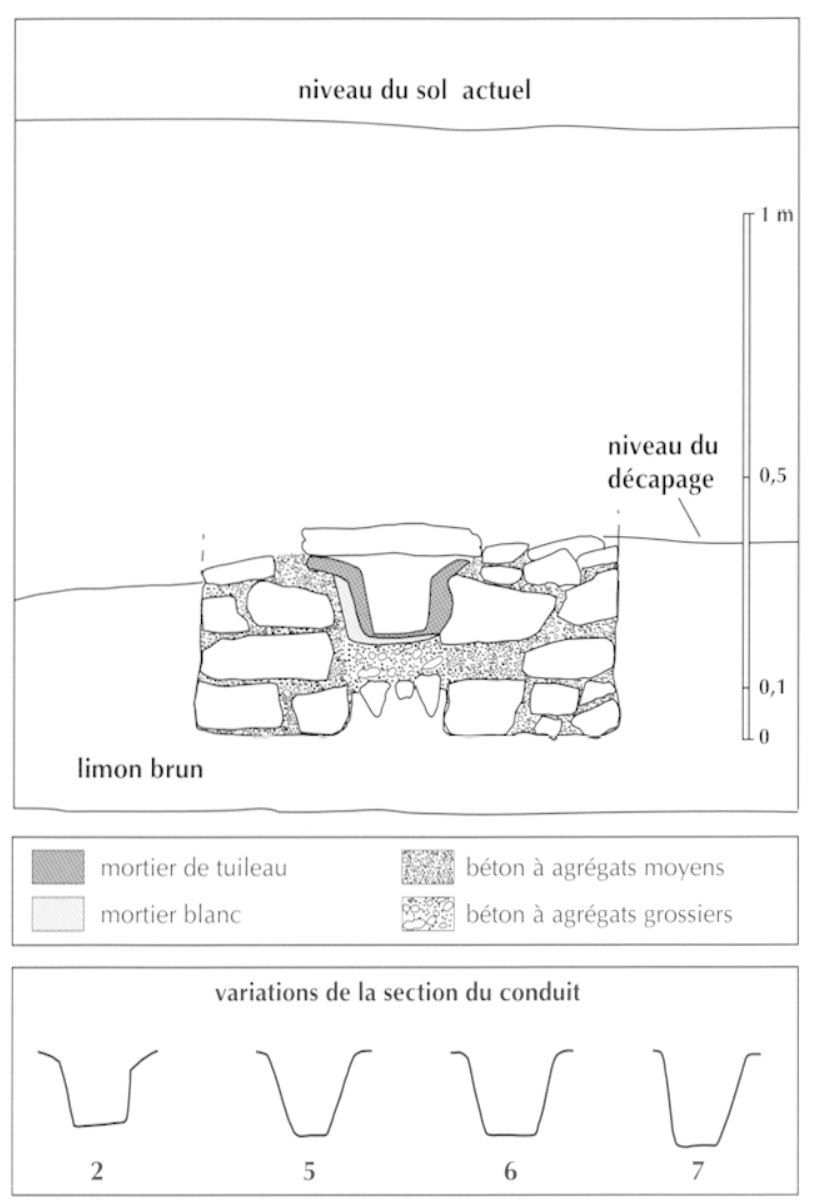

Fig. 101 - Sur Fontorbe à Lavulette et Carcassonne : section de l'aqueduc au point 2 et variations de la section du conduit (I)AO M. Passelac, (CNRS).

La section relevée au point 2 montre la structure de la construction maçonnéc (fig. 101).

Deux piédroits latéraux encadrent le canal. Larges de $0,30 \mathrm{~m}$ à $0,35 \mathrm{~m}$, ils sont constitués de moellons de grès, de poudingue et de molasse (de $0,25 \mathrm{~m} \times 0,15 \mathrm{~m}$ à $0,10 \mathrm{~m}$ $\mathrm{x} 0,10 \mathrm{~m}$ ) liés par un béton de chaux grisâtre à granulats moyens $(5 \mathrm{~mm}$ à $10 \mathrm{~mm})$.

Un radier central, plus léger, supporte le canal (larg., $0,20 \mathrm{~m}$; haut., $0,15 \mathrm{~m})$. Il est formé d'un hérisson de petits moellons irréguliers de grès, haut de $0,07 \mathrm{~m}$, surmonté d'un béton très clair, avec granulats grossiers de graviers et de petits galets $(1.5 \mathrm{~mm}$ à $35 \mathrm{~mm})$.

Le conduit, quant à lui, est maçonné en deux couches successives de béton de tuileau. I a première, de couleur claire, est épaisse de $20 \mathrm{~mm}$ à $30 \mathrm{~mm}$; cllc forme les parois latérales et le fond du conduit. I a seconde, plus riche en tuileau, de couleur rose, épaisse de $30 \mathrm{~mm}$ en moyenne, à la surface soigneusement lisséce, recouvre la précédente et s’étale sur les côtés en un rebord oblique de $0,10 \mathrm{~m}$ de large, sur lequel repose la couverture. I a section du conduit présente des variations de détail (fig. 101). Sa forme générale est trapézoïdale et ses dimensions moyennes sont de $0,10 \mathrm{~m}$ pour la petite base, $0,15 \mathrm{~m}$ pour la grande base, $0,12 \mathrm{~m}$ pour la hauteur et $0,15 \mathrm{~m}$ de hauteur sous couverture.

La couverture est formée de petites dalles de grès, de poudingue et de fragments de legulae de récupération dont les rebords ont été bûchés. Cés matériaux sont posés à plat, jointifs à sec, parfois sur deux épaisscurs pour assurer une meilleure protection contre les infiltrations de limon (fig. 102, $\mathrm{n}^{\text {os }} 1$ et 2 ).

Les deux points les plus éloignés où le fond du conduit a pu être mesuré étaient distants de $36,60 \mathrm{~m}$. La dénivelée mesuréc entre ces deux points était de $0,14 \mathrm{~m}$, soit une pente de $0,38 \%$.

\section{CONTEXTE HYDROGRAPHIQUE}

Le toponyme voisin Fontorbe ${ }^{i 7}$ évoque une source aménagée et protégée par une construction. La source a donné son nom à un important domaine, alimenté au $\mathrm{XX}^{\circ}$ s. par un réservoir d'eau, situé à $250 \mathrm{~m}$ environ au sud de l'aqueduc, sur une éminence. Une station de relevage y refoulait les eaux d'un grand puits creusé en bordure du ruisseau de la Malepère. Nous avons déjà évoqué ce Mairal ou ruisseau de la Malepère, qui coule au sud du domaine de Fontorbe, et la position de l'aqueduc sur la pente d'un vallon très atténué, parcouru lui-même par un petit ruisseau. À quelques dizaines de mètres en aval de la découverte, on note la présence d'un ancien verger, qu'un puits permettait d'irriguer. À $100 \mathrm{~m}$ environ au nord de la conduite, sur le flanc du vallon, à la base d'affleurements de conglomérats, est présent un autre puits, près d'une cabane de vigne. L'eau est donc dans tout ce secteur une ressource abondante, soit par la présence de sources et de puits, soit par l'écoulement dans les ruisseaux sans doute pendant la plus grande partie de l'année.

Cependant, on n'a pas pu localiser le captage alimentant la conduite gallo-romaine. Le puits de Fontorbe et le ruisseau de la Malepère sont situés à une altitude inférieure à celle du tronçon de conduite mis au jour. Aussi faut-il supposer la présence dans l'Antiquité d'une source suffisamment abondante, aujourd'hui disparue, sur un flanc du relief qui domine à l'ouest le site de la découverte.

\section{DESTINATION DE LA CONDUITE}

I a topographic du vallon dicte le tracé de l'aqueduc en aral du point de découverte. Pour conserver une pente suffisante, mais relativement faible, la conduite ne s'éloignait pas beaucoup du tracé de la courbe de niveau des $145 \mathrm{~m}$. Ainsi, peut-on facilement imaginer qu'elle formait un coude, au franchissement du fond du vallon pour se diriger, sur l'autre versant, vers le sud-est. Mais à ce jour, aucun établissement gallo-romain n’a été repéré à proximité de l'aqueduc, en aval. Trois hypothèses se présentent donc quant à la destination de la conduite (fig. 103) :

77. ("est-à-dire la Fontaine murce (occitan : orb, -a : aveugle) (Alibert, 1965, p. 516 ). 

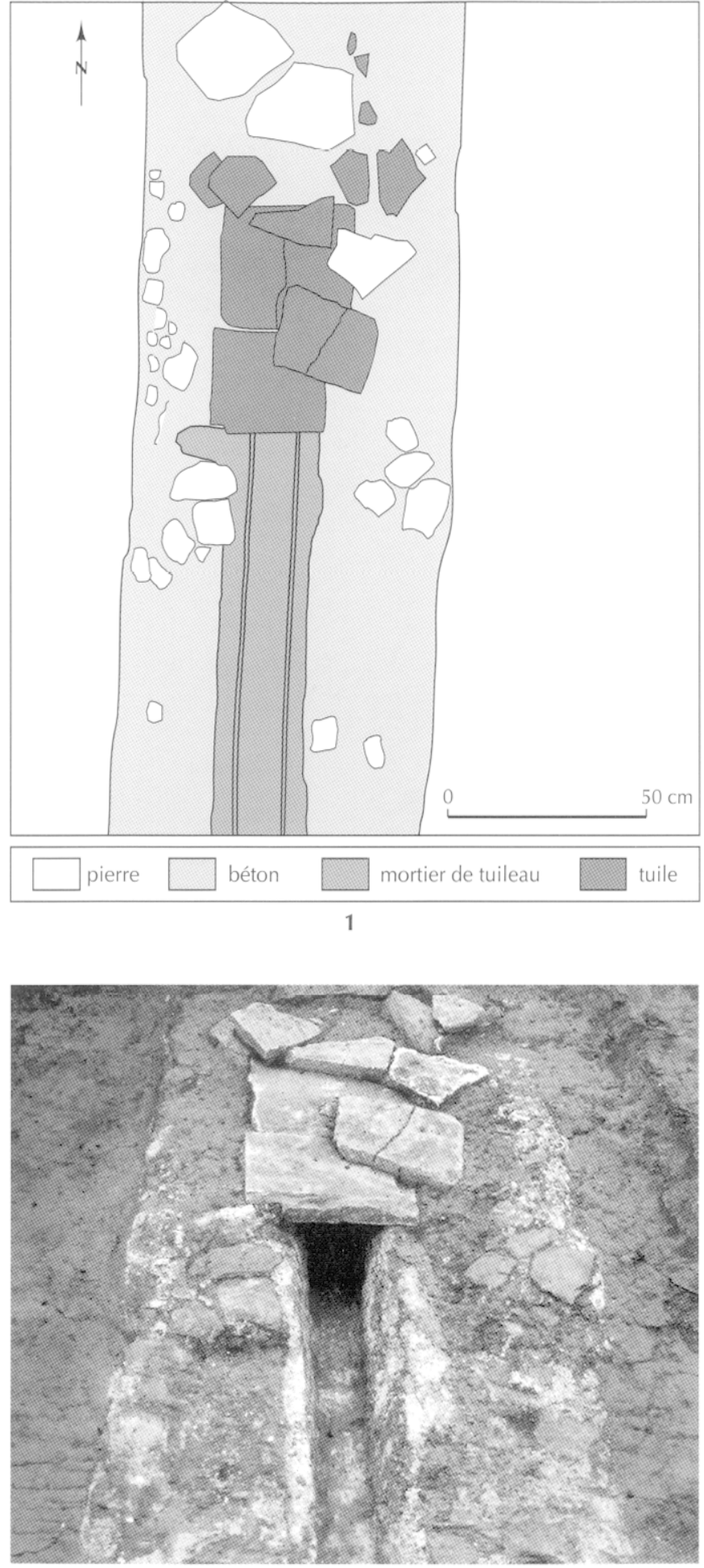

2

Fig. 102 - Sur Fontorbe à Lavalette et Carcassonne: 1, plan de l'aqueduc et de sa couverture entre les points 3 et $4 ; 2$, l'aqueduc vu du sud entre les points 3 et 4 (cliché M. Passelar, CNRS).

- elle alimentait un établissement peu éloigné, mais inconnu à ce jour, peut-être enfoui sous les colluvions et les alluvions comblant le vallon en aval ;

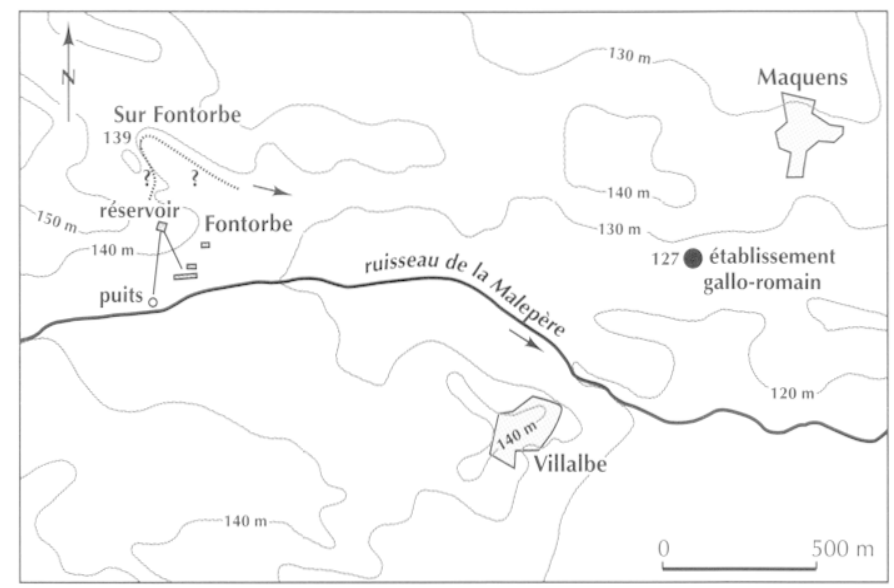

Fig. 103 - Sur Fontorbe à Lavalette et Carcassonne : contexte topographique et hydrographique de l'aqueduc (DAO M. Passelac, CNRS).

- elle alimentait un établissement plus éloigné, comme celui qui a été repéré au sud-ouest de Maquens, aujourd'hui en partie oblitéré par l'autoroute $\mathrm{A} 61^{78}$; cet établissement est situé à $1750 \mathrm{~m}$ du tronçon connu, et à un peu moins de $130 \mathrm{~m}$ d'altitude ;

- elle n'aboutissait pas à un établissement agricole, mais alimentait un réseau d'irrigation en plein champ, dépendant d'un domaine.

\section{CHRONOLOGIE}

Aucun mobilier n'a été rencontré dans les unités stratigraphiques de construction ou de comblement de l'aqueduc. Par ailleurs, les fragments de tegulae utilisés dans la couverture sont trop petits pour donner des indications précises de module et permettre d'approcher ainsi la chronologie de l'ouvrage. Leur épaisseur se situe ente $25 \mathrm{~mm}$ et $30 \mathrm{~mm}$, et peut appartenir aux grandes tuiles du Haut-Empire aussi bien qu'aux tuiles de dimensions plus réduites utilisées au Bas-Empire. De plus, il faut penser que la plupart de ces fragments de tuiles sont très certainement issus de récupérations.

\section{L'AQUEDUC DE BARTISSOL À CASTELNAUDARY (AUDE)}

La connaissance de cet aqueduc est due à la prospection de travaux connexes au remembrement dans la partie sud de la commune de Castelnaudary, en 1980. L'examen de fossés fraîchement creusés de part et d'autre d'un nouveau chemin rural a permis de repérer la présence d'un conduit maçonné et de sa fondation, à proximité d'un établissement gallo-romain déjà connu.

78. Ce site nous a été indiqué par M. A. Bennes, qui le connaissait antérieurement à la construction de l'autoroute. 


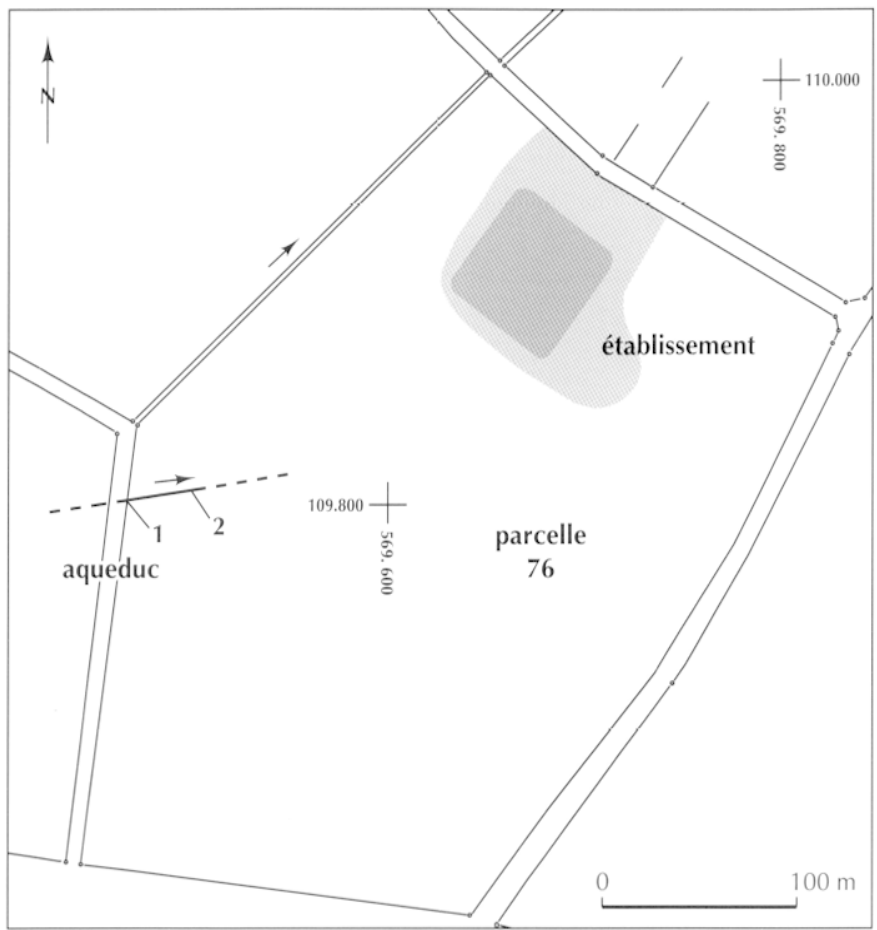

Fig. 104 - Bartissol à Castelnaudary : localisation cadastrale de l'aqueduc et de l'établissement rural (DAO M. Passelac, CNRS).

\section{LOCALISATION DU TRONÇON OBSERVÉ}

L'établissement rural exploitait un secteur de la plaine du Tréboul, à $3 \mathrm{~km}$ au sud-sud-est de Castelnaudary. Sur cette plaine en faible déclivité vers le nord, le site occupe une zone d'alluvions postwürmiennes recouvertes d'un sol peu épais ${ }^{79}$. Le segment de conduite, très court, puisqu'il n'a été observé que dans les parois d'un fossé, est situé à $180 \mathrm{~m}$ en amont de la villa et prend nettement sa direction ou celle de ses abords (fig. 104, $\mathrm{n}^{\circ} 1$ ). Une prospection récente, effectuée en 2003, a permis de confirmer cette destination. Elle a mis en évidence en surface, dans le labour, entre le point de découverte de l'aqueduc et la villa, des matériaux appartenant à la conduite: béton blanc à la chaux et mortier de tuileau (fig. 104, no2).

\section{DESCRIPTION DE LA CONDUITE}

Il s'agit d'une conduite installée dans une tranchée, creusée ici dans le substrat de sables et graviers (fig. 105). Large de $0,50 \mathrm{~m}$ et profonde de $0,18 \mathrm{~m}$ seulement, la tranchée présente la forme d'une cuvette au fond plat et aux parois légèrement concaves. Elle est remplie d'un béton à agrégats grossiers de sables et cailloutis calcaires locaux. Au centre, un conduit de section trapézoïdale est maçonné en mortier de tuileau, épais

79. I a conduite et l'établissement rual sont situés dans la parcelle 76 , section 7H du cadastre de (astehaudary, le site de la villa occupe également la partie sud de la parcelle 77. Carte topographique de l'IGN au $1 / 25000,2245$ ouest, Castelnaudary.

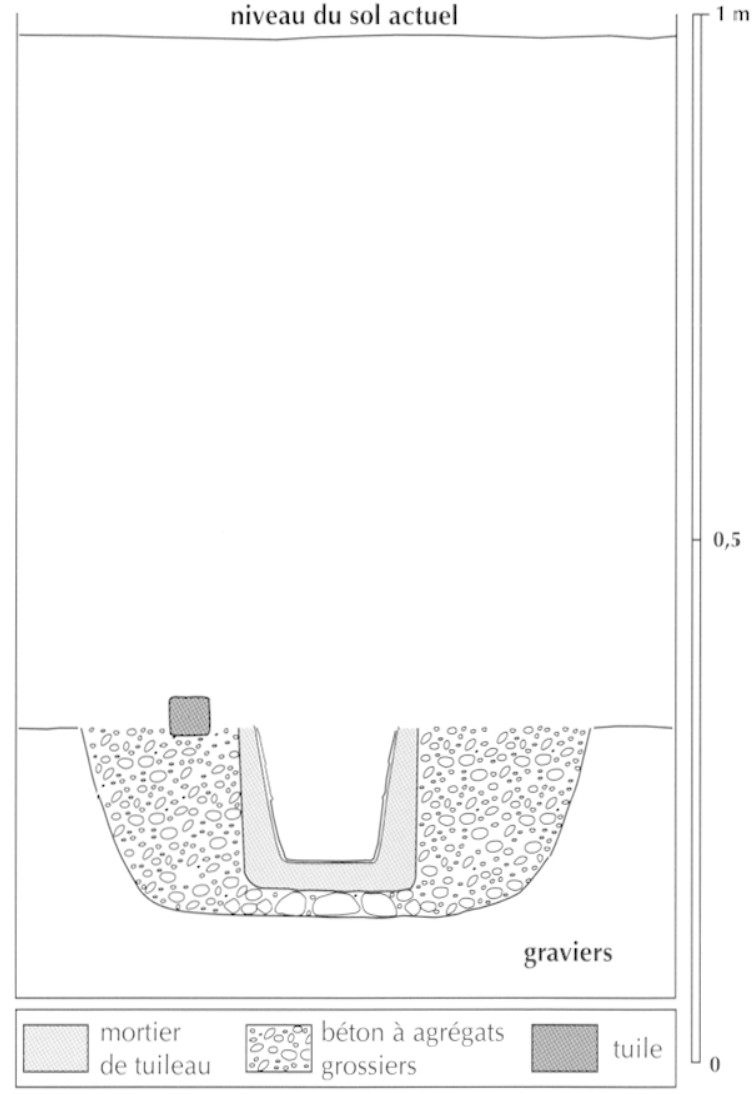

Fig. 105 - Bartissol à Castelnaudary : section dans le fossé de la parcelle 76 de l'aquedur (I)AO M. Passelac; CNRS).

de $10 \mathrm{~mm}$ à $35 \mathrm{~mm}$ pour les parois et de $20 \mathrm{~mm}$ pour le fond. Dimensions intérieures du conduit: petite base, $0,09 \mathrm{~m}$; grande base, $0,14 \mathrm{~m}$; hauteur, $0,14 \mathrm{~m}$. Sur les parois se sont déposées des concrétions calcaires épaisses de $1 \mathrm{~mm}$ dans la partie haute à $5 \mathrm{~mm}$ vers le milieu de la conduite. Un fragment de tegula était encore en place sur le haut de la tranchée, au niveau de l'ouverture du conduit, et témoigne très certainement du mode de couverture de cet aqueduc.

Cette observation très ponctuelle ne permet pas de calculer la pente de la conduite, que nous pouvons tenter de restituer en fonction de son tracé probable et de la pente du terrain selon ce tracé. On supposera que sa profondeur par rapport au sol était constante et que la topographie de la plaine n'a guère évolué sur ce tracé depuis l'Antiquité. Dans l'axe de la conduite, la dénivelée du sol actuel est de $5 \mathrm{~m}$ pour $375 \mathrm{~m}$, soit une pente de $0,13 \%$ à l'approche de la villa.

\section{CONTEXTE HYDROGRAPHIQUE}

Le site est marqué par l'emplacement d'un ancien écoutlement allant des collines de la Piège vers le lit du Tréboul, dépression colmatée par des alluvions postwürmiennes. Aujourd'hui encore, cette zone est traversée par un profond fossé qui matérialise le fond de cette dépression, et un autre ruisseau coule le long du chemin qui, venant de Fendeille, passe près de la Rivière (au nom évocateur) et se dirige vers le 
Sergentou: sur le cadastre de $1823^{81}$, il porte le nom de ruisseau de Bartissol. Le site de la villa est encadré par ces deux petits ruisseaux. Il est propice, en outre, à l'approrisionnement en eau par des puits.

Le captage à l'origine de cet aqueduc n'est pas précisément connu. L'aqueduc pouvait capturer l'eau des ruisseaux passant à l'ouest de la villa, à quelques centaines de mètres en amont de celle-ci (fig. 106). On doit également évoquer la découverte d'un petit bassin en béton de tuileau par un agriculteur, dans les années 1960, lors du curage de fossés, au nord de la ferme la Planète. Cette construction, qui paraissait isolée, ne peut plus être aujourd'hui localisée avec précision, mais sa technique de construction et sa situation approximative par rapport au point de découverte de l'aqueduc permettent d'y voir le captage de départ. Dans ce cas, il aurait une longueur d'environ $850 \mathrm{~m}$.

\section{DESTINATION DE LA CONDUITE}

La présence d'un établissement gallo-romain à $180 \mathrm{~m}$ du tronçon reconnu et l'orientation de l'aqueduc ne laissent en revanche aucun doute sur sa destination, même si on ne sait s'il aboutissait aux abords ou au coeur même de l'établissement.

Celui-ci est relativement important. L'épandage des matériaux qui le matérialisent couvre environ $8000 \mathrm{~m}^{2}$, mais la zone de forte concentration où se rencontrent des moellons de calcaire blanc, de marne, de grès, et des tuiles, qui correspond à la partie bâtie, s'étend sur unc superficie de 4000 à $5000 \mathrm{~m}^{2}$.

\section{Chronologie}

La chronologic de l'aqueduc ne peut être précisée par l'observation de la structure elle-même. Aucun artefact n'a été retrouvé dans la construction ou le comblement de la conduite observée de façon très ponctuelle. En revanche, la datation de l'établissement permet de proposer une fourchette à l'intérieur de laquelle s'insèrent la construction et le fonctionnement de cette conduite. Les mobiliers recueillis en surface sur le site indiquent une occupation qui couvre la période républicaine et le Haut-Empire. Les fragments d'amphores italiques, de dolium indigène, de céramique non tournée appartiennent à un tablissement précoce, comme il en existe beaucoup dans cette plaine (Passelac, 1994), mais on peut éliminer d'emblée cette période pour la datation de l'aqueduc, dans la mesure où l'usage de la chaux était à ce moment-là inconnu dans cette région. Les mobiliers d'époque gallo-romaine n'ont pas été recueillis en grande quantité, mais semblent se circonscrire au $\mathrm{I}^{\mathrm{Cr}}$ s. apr. J.-C. : fragments d'amphores Pascual 1, Dressel 2-4, Dressel 28, Dressel 20, céramique sigillée de Ia Graufesenque du I ${ }^{\mathrm{er}}$ s. apr. J.-C. et probable as de Claude. Dans l'état actuel de nos données, la conduite paraît donc appartenir à cette deuxième phase de l'occupation du site qui est assez. tôt abandonné et ne présente pas, malgré sa forte taille, le caractère d'unc riche résidence. Les prospections n'ont pas livré de restes de marbres, de mosaïques, de sols bétonnés qui caractérisent les villa opulentes et leurs parties thermales présentes dans la région, le plus souvent à une période plus tardive.

80. Ce document est conservé à la mairie de Fendeille (Aude).

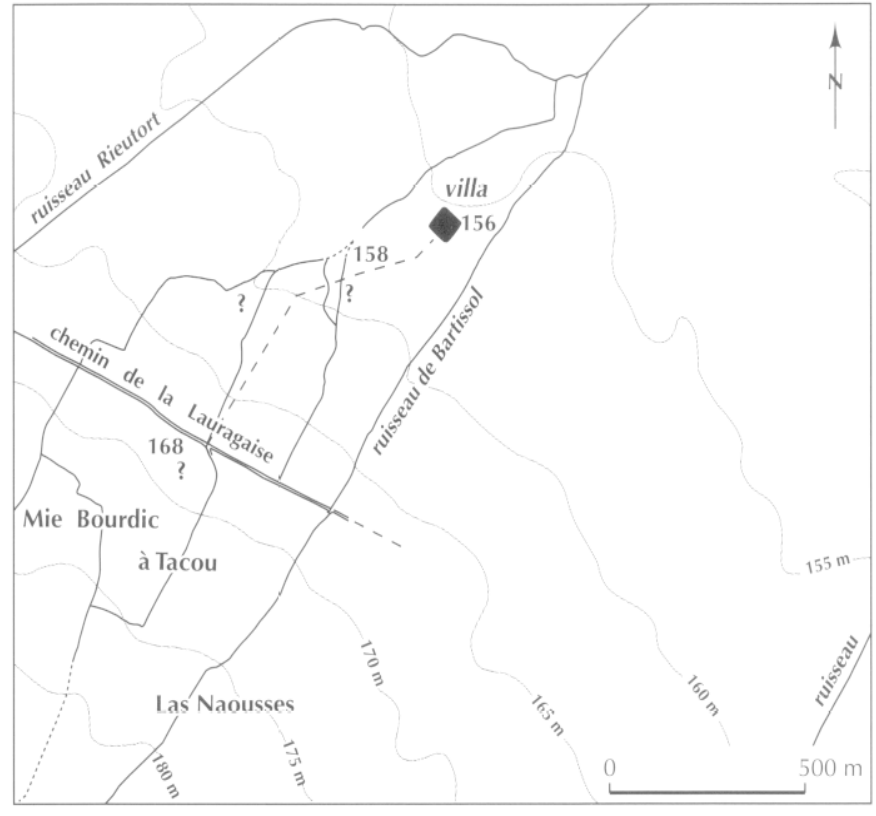

Fig. 106 - Bartissol ì Castelnaudary : contexte topographique et hydrographique de l'aqueduc, d'après le cadastre de 1823 (I)AO M. Passelac, (NRS).

\section{L'AQUEDUC DE LA BARONNE À MAS-SAINTES-PUELLES (AUDE)}

Cette conduite a été repérée, encore une fois, par la surveillance de travaux de terrassement. Il s'agit ici de la pose d'un gazoduc de la Société nationale des Caz du Sud-Ouest en 1983. Nous avions assuré une prospection de surface du tracé de ce gazoduc avant la réalisation du chantier, puis un examen attentif des tranchées au moment des travaux ${ }^{81}$. Cette deuxième prospection a livré des vestiges que nous interprétons comme ceux d'un aqueduc souterrain.

\section{LOCALISATION DU SEGMENT DE CONDUITE}

Ces vestiges sont apparus sur les deux faces de la tranchée de pose du gazoduc au lieu-dit La Baronne, commune de MasSaintes-Puelles ${ }^{82}$ (fig. 107). Le site de la découverte se localise, comme celui de la conduite précédente, dans la vallée du Tréboul, dans cette large plaine qui s'étend des collines de la Piège au sud, jusqu'au cours du ruisseau. Le sous-sol y est formé d'alluvions würmiennes: graviers hétérogènes et sables argileux. Près de la conduite, à l'cst, apparaissait le substrat molassique ludien (BRGM, 1975).

81. Cette opération avait été organisée avec l'autorisation et l'aide matérielle du Service régional de l'archéologie de I anguedoc-Roussillon, et avait bénéficié de la collaboration de J.-P. Cazes.

82. La tranchée du gazoduc longeait l'autoroute A61 dans la parcelle 17 section ZK du cadastre de Mas-Saintes-Puclles. I es vestiges ont été rencontrés à $31 \mathrm{~m}$ de la limite est de la parcelle. Carte topographique de l'ICX au 1/25000, 2245 ouest, Castelnaudary. 


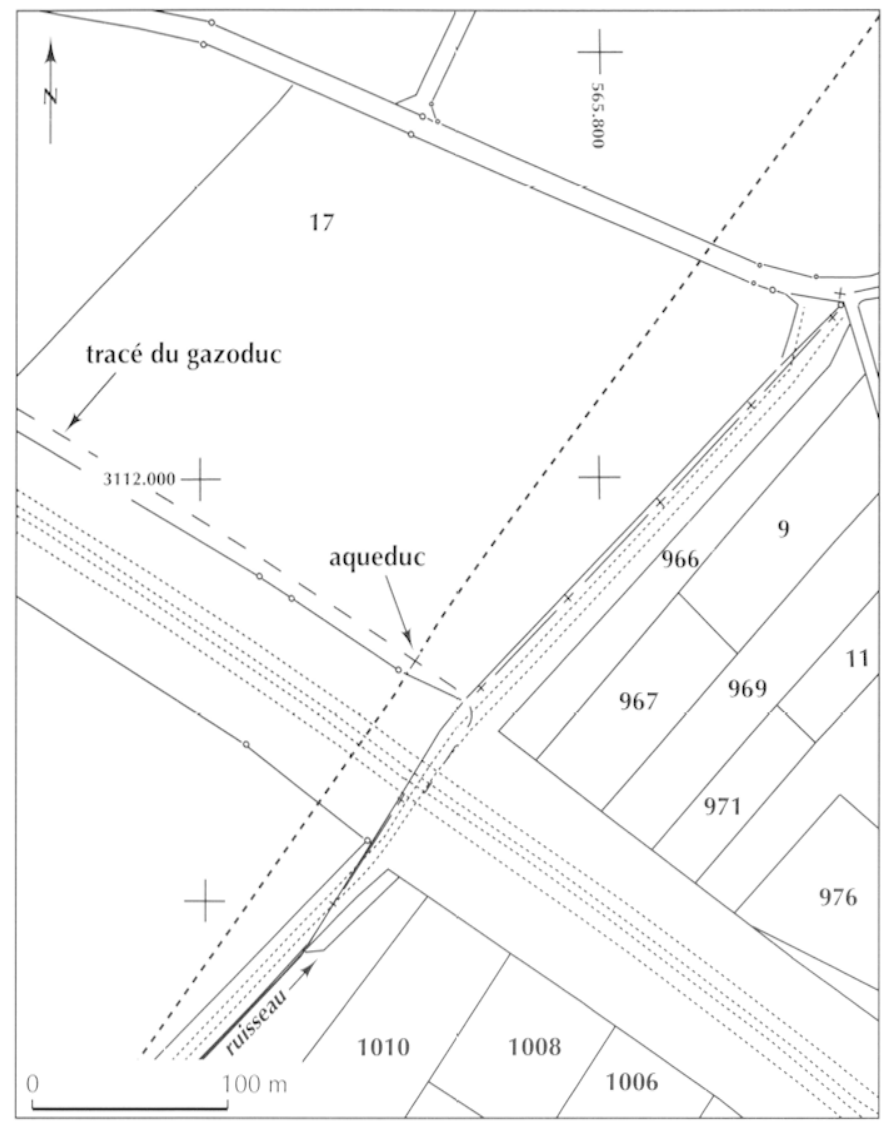

Fig. 107 - La Baronne au Mas-Saintes-P’uelles : localisation cadastrale du point de découverte de l'aqueduc (I)AO M. Passelac, (NRS).

Le point de découverte se situe à une distance de $575 \mathrm{~m}$ à l'amont d'un important établissement gallo-romain, la villa de Bernardis, découverte dans les années 1960, puis connue par des sondages effectués par A. Monod (Barruol, 1969) et deux opérations de fouilles préventives en 1988-1990 et 1992 (Passelac, 1993).

\section{DESCRIPTION ET INTERPRÉTATION DES VESTIGES}

Sur une largeur de $3 \mathrm{~m}$ environ, plusieurs creusements sont apparus entre $0,60 \mathrm{~m}$ et $1,40 \mathrm{~m}$ sous le sol actuel. Bien que proches, les faces nord et sud de la tranchée ne donnent pas une image strictement identique des comblements (fig. 108), mais on peut restituer l'histoire de ces terrassements anciens.

La succession de creusements et de comblements dans cet espace asse\% restreint, linéaire, est complexe. I.c creusement 2005 s'identific asse\% facilement à un fossé ayant amené de l'eau en raison de la présence d'une couche de sables et de graviers à sa base. Celui du creusement 1013-2015 est plus curicux; c'est son comblement, déposé sur le fond (US 1012-2013), qui permet de proposer l'hypothèse d'un aqueduc épicré. Il renferme en effet des petits mocllons, des restes de mortier de chaux, des restes de mortier de tuileau,
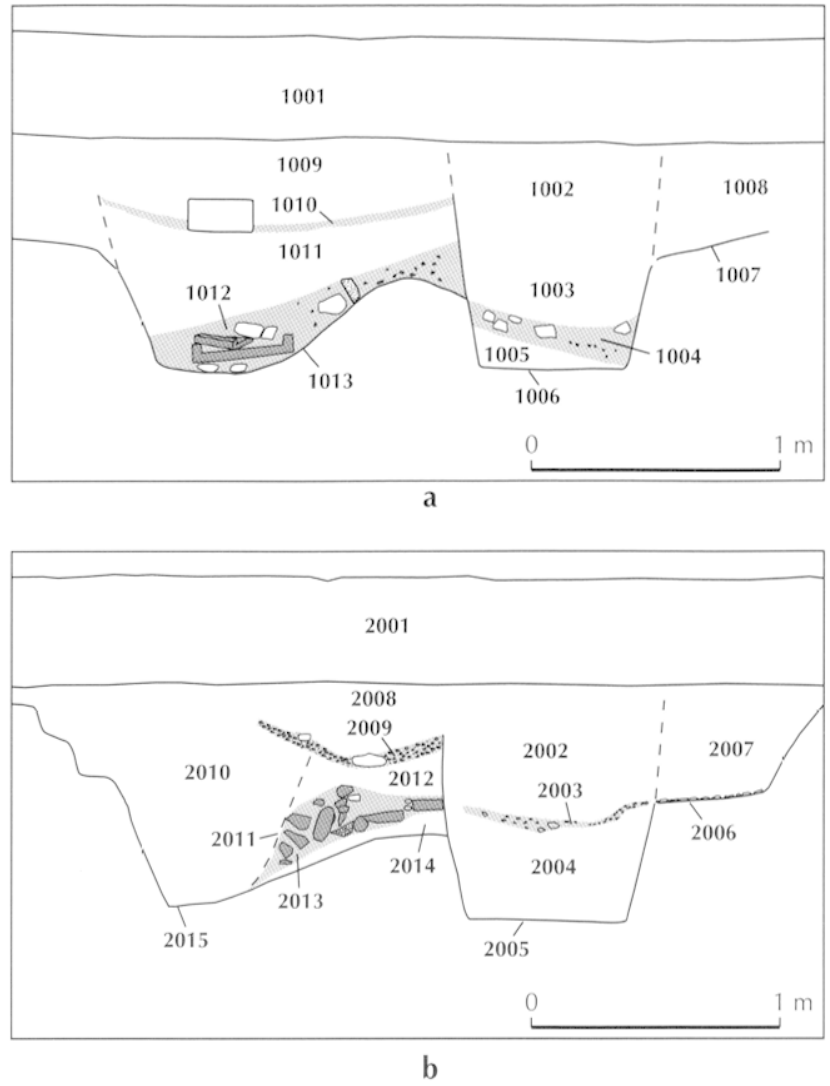

Fig. 108 - La Baronne au Mas-Saintes-Puelles : sections de l'épierrement de l'ouvrage : a, au nord de la tranchée du gazoduc; b, au sud de la tranchée du gazoduc (inversée) (I)AO M. Passelac, (NRS).

des gros fragments de tegulae, voire des tegulae presque entic̀res, rejetés pêle-mêle lors du remblaiement du creusement, type de comblement que l'on observe généralement dans les tranchées d'épierrement. Si l'on prend comme modèle l'aqueduc de Sur Fontorbe, nous avons ici tous les matériaux utilisés dans la construction de la conduite, à l'exception des moellons de grande taille ou de taille moyenne, qui ont été récupérés ${ }^{83}$ : moellons et mortier de chaux des murets de fondation, béton et mortier de tuileau de la conduite, fragments de tegulae utilisées pour la couverture. Cette hypothèse est renforcée par le creusement du côté conservé de la paroi en marches d'escalier, dispositif probablement destiné à faciliter l'accès à la partie basse de la tranchée pour la mise en ceure de matériaux lors de la construction.

Après cette phase de récupération des matériaux, plusicurs creusements ont été opérés au même endroit, dont un semble ponctuel (2010). Ĺne légère dépression a subsisté, dont le fond est matérialisé par les LS 1010 et 2009. Eille a été combléc plus tard par des limons. Enfin, un fossé (1006-2005) constituc le creusement le plus récent puisqu'il recoupe presque toute la

83. Un moellon taillé est cependan présenn dans l'US 1010, mais celleci est postéricure, semble-t-il, à l'épierrement. 


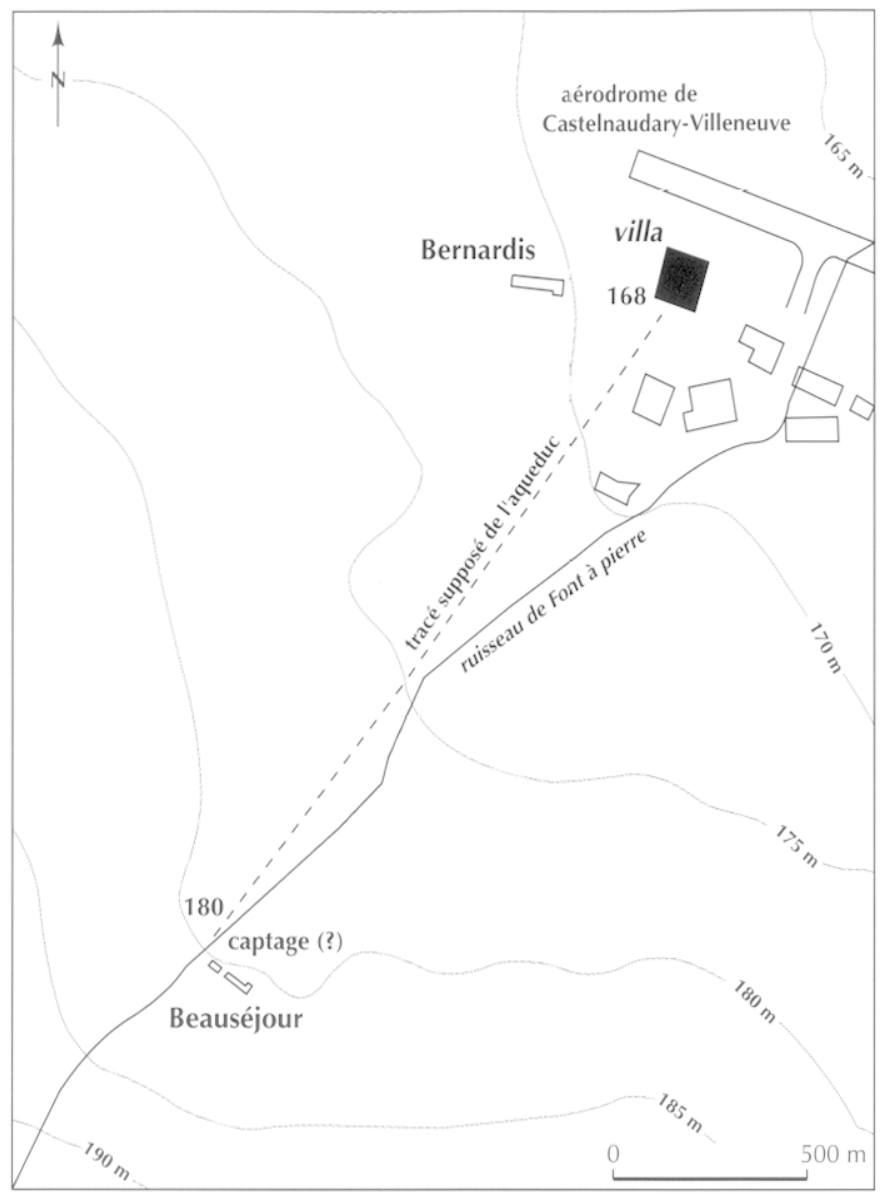

Fig. 109 - Mas-Saintes-Puelles, aqueduc de La Baronne et villa de Bernardis dans leur contexte lopographique et hydrographique (IDAO M. Passelac, (NRS).

stratigraphie. Sa phase d'activité témoigne, on l'a souligné, d'une fonction d'écoulement des eaux.

\section{CONTEXTE HYDROGRAPHIQUE}

Le point de découverte de cet aqueduc épierré se situe, dans la vallée du Tréboul, sur le versant d'une légère dépression qui a son origine non loin du piémont des collines et se dirige vers le nord en s'ouvrant, perpendiculaire au cours du Tréboul (fig. 109). À la tête de cette dépression, près de la ferme de Beauséjour, vers $180 \mathrm{~m}$ d'altitude, nous avons observé dans le chantier d'un nouveau gazoduc, en 1996, un thalweg colmaté par des sédiments très organiques et gorgés d'eau. Il y avait sans doute là un ancien écoulement qu'aujourd'hui encore un puits met à profit. Cette dépression alimentée par un large bassin versant a également déterminé l'emplacement d'un passage sous l'autoroute A61, qui associe une voie carrossable et un passage des eaux. Quelques centaines de mètres au nord, la partie basse de la plaine nécessite de forts fossés de drainage qui, au-delà de la piste de l'aérodrome, se déversent dans le Tréboul. L'aqueduc pressenti se situe donc en aval d'un contexte topographique favorable à l'accumulation de l'eau et

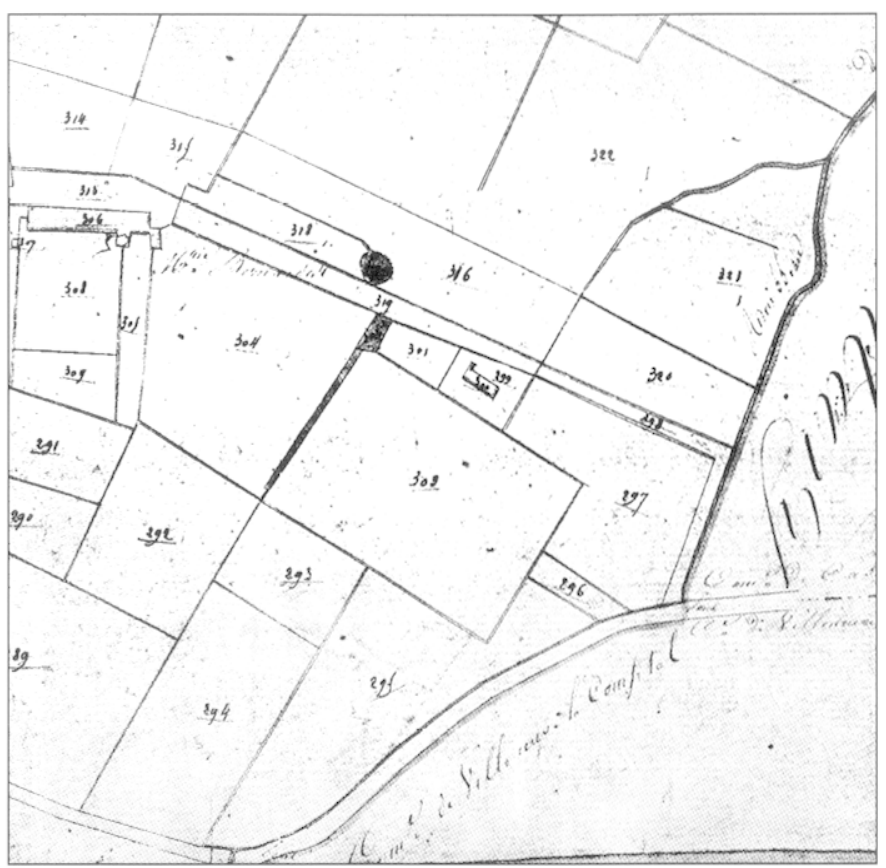

Fig. 110 - Site de Bernardis, mares et ruisseaux (d'après le cadastre de Mas-Saintes-Puelles en 1823).

à proximité immédiate d'une ligne naturelle d'écoulement de celle-ci. L'examen du cadastre de 1823 porte le tracé de ce ruisseau et le nomme ruisseau de Font à picrre ${ }^{84}$. Cc document situe la source au sud-ouest de la ferme de Bordeneuve et montre le passage du ruisseau à proximité immédiate du site de la villa de Bernardis où il a été rectifié en deux branches dont une alimente deux mares (fig. 110).

\section{DESTINATION DE LA CONDUITE}

La direction prise par les tranchées et le fossé observés est celle de l'important établissement rural de Bernardis situé à un peu moins de $600 \mathrm{~m}$ au nord et à $168 \mathrm{~m}$ d'altitude. Le site est occupé dès l'âge du Bronze final (Passelac, 1993). Des vestiges de la période républicaine ont été retrouvés à proximité immédiate et à l'emplacement de la villa qui semble construite dans la première moitié du $\mathrm{I}^{\mathrm{er}} \mathrm{s}$. apr. J.-C. Elle est dès lors occupée jusqu'au Bas-Empire, dans le courant du ve $s$. Cependant, la présence sur les lieux d'un cimetière de la période mérovingienne, qui a livré notamment une remarquable plaque-boucle burgonde, atteste la continuité de l'occupation. On y situe au Moyen Âge le site ecclésial et décimaire de Saint-Pierre-de-Campagne (Cazes, 1998, p. 525).

Dès le Haut-Empire, l'établissement cst important: il couvre déjà probablement plus de $3000 \mathrm{~m}^{2}$. Les parties reconnues pour cette période sont un grand bâtiment de plan basilical, peut-être un chai, et un autre bâtiment agricole de fortes dimensions limité au sud par un mur à contreforts (Passelac, à paraître). Dans le courant du $\mathrm{II}^{\circ} \mathrm{s}$., une réorgani-

84. Sections C.3 et B3 du document conservé à la mairie de Mas-SaintesPuelles (Aude). 
sation importante transforme cette partie en une vaste aile thermale qui couvre environ $200 \mathrm{~m}^{2}$ et subit plusieurs remaniements. Depuis sa construction, la villa est un grand établissement agricole et le soin apporté au confort de ses occupants en fait une riche résidence au Bas-Empire.

Les observations réalisées au cours des deux opérations de fouille préventives ont montré que l'établissement était situé sur un très léger relief en bordure d'un petit ruisseau, celui-là même qui coulait en provenance des environs de Bordeneuve. Il alimentait très probablement une mare observéc lors de la construction d'un bâtiment de l'usine aéronautique; aménagement daté du $I^{\text {er }} \mathrm{s}$. av. J.-C. et situé à un emplacement très proche des mares du XIX"s.

\section{DES TECHNIQUES DE CONSTRUCTION PARTICULIÈREMENT SOIGNÉES}

Malgré leurs dimensions très réduites, ces conduites ont été réalisées avec des techniques de construction élaborées, adaptées aux exigences du transport de l'eau et aux contraintes que pouvaient subir ces ouvrages. En réalité, à une échelle réduite, la morphologie de l'aqueduc de Sur Fontorbe présente les mêmes caractéristiques que celles des aqueducs de grand module, alimentant les cités. Le schéma général est identique, avec deux piédroits maçonnés, un radier de béton, en plusieurs couches, reposant sur un hérisson de petits moellons, un revêtement de béton de tuileau, également en plusieurs couches, soigneusement lissé pour faciliter l'écoulement de l'eau, formant un retour " en crosse " qui caractérise le specus de certains grands ouvrages, comme celui de Traslay qui conduit l'eau à Avaricum/Bourges (Adam, 1984, fig. 570). On rapprochera également notre section de celle des aqueducs du Mont d'Or et d'Izernon, qui alimentaient Lyon (Grenier, 1960, fig. 39 ; Burdy, 2002, p. $81-83$ et fig. 38 et 39). Pour ce qui concerne les adductions plus modestes, l'aqueduc de Villedieusur-Indre (Indre) offre une section proche de nos conduites, mais le specus est rectangulaire (Ferdière, 1988, p. 230). On peut penser que la solidité de la conduite de Sur Fontorbc a ćté rendue nécessaire par la nature du terrain parcouru à l'endroit de la découverte. S'agissant de limons, susceptibles de se tasser, il était impératif d’asseoir la conduite sur une fondation large et rigide. Les moellons utilisés pour cette fondation étaient asse $z$ facilement prélevés dans l'environnement proche du chantier, constitué de molasses et poudingues du Lutétien (BRGM, 1977).

L'aqueduc de Bartissol présente une structure moins massive et plus simple, car il est établi dans un contexte géologique différent. Le substrat de sables et graviers dans lequel la tranchée a été creusée offrait une assise d'une stabilité parfaite, et les matériaux extraits pouvaient être immédiatement recyclés dans le béton de la fondation. Les parois du conduit sont de la même forme et de la même technique que celles de l'ouvrage de Sur Fontorbe.

La technique de construction de l'aqueduc de La Baronne, épierré, n’a pu être observée, mais d’après la nature des matériaux résiduels dans la tranchéc, on peut penser qu'clle s'apparentait à celle utilisée dans le cas de Sur Fontorbe.
Ajoutons que les travaux de nivellement, qui devaient être très précis pour garantir une pente adaptée, nécessitaient des compétences particulières. Celles-ci étaient cependant moins développées que celles qu'exigeaient les grands ouvrages dont la longucur multipliait les difficultés rencontrées (Fabre $e t$ al. dir., 1991). Ainsi, ces conduites rurales de longueur restreinte, parcourant des profils topographiques simples, ne présentaient pas de tunnels, de siphons ni, semble-t-il, de regards, puisque leur couverture, sans doute proche de la surface, pouvait être soulevée en cas d'obstruction.

Au total, on retiendra que la réalisation de ces conduites devait constituer un investissement non négligeable en nivellement, transport et mise en ceuvre de matériaux $\left(1 \mathrm{~m}^{3}\right.$ pour $3 \mathrm{~m}$ linéaires à Sur Fontorbe, soit $80 \mathrm{t}$ environ pour $100 \mathrm{~m}$ linéaires). F́tait-elle confiée à des entreprises spécialisées?

\section{D'IMPORTANTS BESOINS EN EAU}

Le calcul du débit de ces conduites peut nous permettre d'approcher le volume des besoins en eau et d'émettre des hypothèses quant à l'utilisation de cette eau. Il s'agit bicn évidemment d'une approche, car les inconnues sont importantes. On ne connaît pas, en effet, la hauteur de remplissage de la conduite et ses variations. On ignore, en outre, si la source ou le ruisseau alimentant les aqueducs coulait toute l'année ou seulement de façon périodique.

C'est encore l'aqueduc de Sur Fontorbe qui va nous servir de référence, car il fournit les données les plus complètes.

Nous avons calculé son débit potentiel en fonction de la moyenne des sections observées, soit $0,02 \mathrm{~m}^{2}$ et de la pente mesuréc sur le tronçon mis au jour, soit $0,0038 \mathrm{~m}$. Le calcul a été effectué selon la formule de Bazin, en retenant un coefficient de rugosité $(\gamma)$ de 0,16 puisque les parois étaient lisses et ne présentaient aucune concrétion. Les résultats obtenus se trouvent dans le tableau VII.

D'une section comparable, l'aqueduc de Bartissol pouvait avoir un débit équivalent, sans doute même supérieur, si sa pente était bien identique à la pente du terrain, soit 0,013 .

Les dimensions de l'aqueduc de Bernardis ne sont pas connues, mais on peut raisonnablement penser qu'elles ne sont pas inférieures à celles des deux autres conduites et que son débit était comparable.

Ces quantités d'eau transportée, même en ne retenant que les chiffres les plus bas, paraissent asse $\%$ considérables pour un établissement privé et dépassent très largement les quantités d'eau disponibles dans les villes. Celles-ci sont évaluées par J.-P. Adam à $540 \mathrm{l} / \mathrm{j}$ par habitant à Pompéi, et $1100 \mathrm{l} / \mathrm{j}$ par habitant à Rome (Adam, 1984, p. 268). Ainsi se pose la question de l'utilisation de cette eau courante qui vient s'ajouter aux autres possibilités d'approvisionnement: puits et ruisseaux dans les cas de Bartissol et de Bernardis.

Sans doute l'eau courante est-elle prisée pour la boisson car le mouvement qui l'anime la rend plus saine. Mais la boisson des hommes et l'abreuvement des animaux, fussent-ils très nombreux sur le domaine, ne peuvent justifier à eux seuls l'apport de telles quantités. Dix litres environ par porc de $100 \mathrm{~kg}$, deux ou trois dizaines de litres par bovin et ćquidé 
Tabl. VII - Aqueduc de Sur Fontorbe : tébit et quantité d'eau transportée en fonction du remplissage de la conduite.

\begin{tabular}{|l|c|r|c|}
\hline Écoulement & Hauteur de l'eau $(\mathrm{m})$ & Débit $(\mathrm{I} / \mathrm{s})$ & Quantité transportée/jour $\left(\mathrm{m}^{3}\right)$ \\
\hline maximum & 0,15 & 13,40 & 1157 \\
\hline moyen & 0,10 & 7,00 & 605 \\
\hline bas & 0,05 & 2,47 & 386 \\
\hline
\end{tabular}

adultes, nécessitent quotidiennement tout au plus quelques mètres cubes. Ceux-ci peuvent être facilement trouvés dans des mares alimentées par les fossés, les ruisseaux et la récupération des eaux de pluie venant des toitures. De telles mares ont été observées en Lauragais sur les villae de l'Estrade à Mireval, sur celle de Pech d'L'ssaud à Castelnaudary (Passelac, 2000) et celle de Bernardis, comme on vient de le voir. Sur la ferme précoce de Co de Roque à Fendeille, une probable mare est révélée par la photographie aérienne (Passelac, 1994). C'est ainsi que le bétail était abreuvé dans les exploitations agricoles de la région jusqu'au milieu du $\mathrm{XX}^{\mathrm{e}} \mathrm{s}$.

Dans le cas de la villa de Bernardis, l'important quartier thermal a pu bénéficier de l'eau apportée par l'aqueduc. On a considéré en effet qu'en Gaule la plupart des aqueducs privés étaient liés au fonctionnement de balnéaires (Ferdière, 1988, p. 228-229). Mais dans ce domaine, on a montré depuis que les besoins sont relativement limités, car ils pouvaient aussi être satisfaits par la récupération des eaux de pluie ou le pompage dans des puits (Bouet, 1999).

D'autres hypothèses nous paraissent donc devoir être évoquées pour justifier la mise en place de pareils équipements dans notre région.

L'utilisation de la force motrice. Elle paraît difficile dans un pareil contexte topographique sans aménagements particuliers. En effet, en site de plaine, on ne dispose pas de dénivelée suffisante pour faire mouvoir un moulin avec un faible débit. Il est nécessaire de constituer une importante réserve d'eau, dans un réservoir surélevé, pour la libérer au moment d'actionner les meules. De plus, ce stockage a dû nécessiter des installations de relevage par roues ou pompes. Une telle installation est envisagée pour la villa de Montmaurin, Haute-Garonne (Fouet, 1969, p. 145-146), mais nous n'avons aucune donnée précise sur des sites très arasés comme Bartissol et Bernardis.

L'irrigation des cultures vivrières et des jardins d'agrément. C'est dans ce domaine que les besoins en eau pouvaient être les plus importants. En effet, les cultures de plein champ pratiquées dans l'Antiquité, céréales, vigne, légumineuses, ne nécessitent pas, dans le contexte climatique de cette région, un apport d'eau complétant les précipitations naturelles qui sont en moyenne de $720 \mathrm{~mm}$ par an à Castelnaudary. Cependant, leur répartition au cours de l'année est très irrégulière, marquée par une forte pluviométrie printanière. Le climat local est encore caractérisé par une brusque période chaude et sèche au début de l'été, accentuée par des vents desséchants, qui précipite la maturation en fin de végétation (Collomb, 1985 ; Piat, 1985, p. 7-9). Aujourd'hui, le recours à l'irrigation est seulement pratiqué en Lauragais pour des cultures d'été à forte valeur ajoutéc et pour des espèces d'introduction récente, nécessitant un apport spécifique en eau (maiss, sorgho, tomate, haricot...).

Dc même, l’irrigation des prés, pour l'alimentation du cheptel de labour, ne parait pas ici d'une nécessité prégnante. Les terres situées sur les sols limoncux, près de ruisseaux, pouvaient certainement fournir une herbe suffisante. On en trouvait près de Bernardis, en bordure du Tréboul et du ruisseau de Font à pierre, $\mathrm{ct}$, au sud de Bartissol, le toponyme les Naouses (occitan : nausa) évoque bien des prairies humides (Alibert, 1965, p. 506). Près de Fontorbc, les terrains limoneux des bords du ruisseau de la Malepère étaient également propices à la croissance de l'herbe.

En revanche, on peut supposer que dans l'Antiquité les cultures vivrières mises en place aux abords des établissements, ainsi que les vergers, pouvaient bénéficier d'une petite irrigation gravitaire, probablement distribuée à la raic. Si cet apport n'était pas strictement indispensable en dehors de la période sèche, il pouvait garantir, par une mise en cuvre au début de l'été, en juin et juillet, une régularité des résultats. À cette période de l'année, les sources ou les ruisseaux coulent encore avec un débit suffisant. C'est sans doute à la petite irrigation qu'était consacrée une grande partic de l'cau amenée par ces aqueducs privés.

Les jardins d'agrément constituaient le cadre naturaliste des maîtres des maisons urbaines, comme des villae rurales. Aux jardins intérieurs de Pompéi (Bulleri, 2001, p. 17-23) répondent les jardins, intérieurs ou extérieurs, des établissements du midi de la Gaule comme ceux de Montmaurin (Fouet, 1969, p. 129-132). Ces jardins nécessitaient un apport non négligeable d'eau, non seulement pour entretenir la végétation qui les ornait, faire croître les plantes aromatiques et médicinales, mais aussi pour alimenter les fontaines, bassins, vasques qui les animaient et les rafraichissaient (Grimal, 1984).

Dans l'ouest du territoire de la cité de Carcassonne et à ses abords, certains éléments nous permettent d'évoquer la présence de l'eau, probablement de l'eau courante, dans les cours, les jardins et les espaces ouverts des villce. Ainsi, la photographie aérienne a-t-elle révélé un bassin circulaire au centre de la cour intérieure de la villa de Cantarrane à Avignonet-Lauragais ${ }^{85}$. Sur le site de la villa du Souleiha (commune de Castelnaudary), nous avons recueilli en surface un gros fragment d'une vasque en marbre de plus de $1 \mathrm{~m}$ de diamètre (fig. 111). Dans l'aile sud-ouest du bâtiment résidentiel de la villa de Flassian à Limoux, en bordure de la cour, ce sont les vestiges d'une fontaine qui ont été identifiés, et le jardin était agrémenté d'un bassin circulaire (Rancoule, 1988 , p. 34).

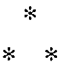

Les trois aqueducs découverts lors de travaux dans l'ouest audois nous éclairent sur l'approvisionnement en eau de certaines villae gallo-romaines de l'Aude. Situés dans une région de transition entre les climats océanique et méditerranéen, relativement bien arrosée, ces établissements pouvaient disposer en même temps d'autres sources d'approvisionnement

85. Prospection aérienne, inédite, de M. Passelac et J.P. Cazes, du 18 juin 1986. 


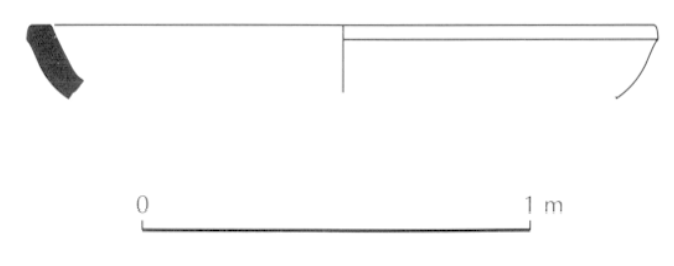

Fig. 111 - Fragment de vasque en marbre de la villa du Souleiha à Castelnaudary (IDAO M. Passelac, CNRS).

en eau: puits et petits ruisseaux. Ces petits aqueducs, très soignés, captaient l'eau d'une source ou d'un ruisseau à quelques centaines de mètres des établissements, sans doute sur les terres du domaine. Au prix d'un investissement en compétences et en travail non négligeable, ils apportaient une quantité supplémentaire et importante d'eau courante de bonne qualité utilisée, on l'imagine aisément, aux besoins multiples de l'exploitation. Mais le volume disponible quotidiennement couvrait très largement les besoins domestiques. Aussi peut-on penser que la majeure partie de cette eau était utilisée à l'irrigation de cultures vivrières et fruitières notamment, au moment où les conditions climatiques l'exigent, c'est-à-dire au début de l'été et, de façon plus permanente, à l'arrosage, à l'animation des jardins d'agrément.

L'absence de fouilles extensives de villae gallo-romaines limite notre connaissance de l'approvisionnement en eau des établissements de cette région. Ces conduites pourraient être cependant un équipement assez fréquent dans l'ouest de la cité de Carcassonne, témoignant de l'optimisation des ressources naturelles par l'aménagement du territoirc. Au-delà des trois exemples que nous venons d'étudier, on peut soupçomner la présence de conduites similaires sur la villa de
Mouriès à Villeneuve-la-Comptal, où une photographie aérienne révèle une structure linéaire partant de la source du Roc vers cet établissement, sur la villa de Flassian à Limoux (Rancoule, Roger, 1994, p. 6), sur la villa d'Al Rec à Fendeille, où le ruisseau évoqué par le toponyme a pu être capturé au profit de l'établissement et sur bien d'autres sites situés en bordure de ruisseaux ou non loin de sources abondantes.

On rapprochera ces aqueducs aux conduits maçonnés, de section trapézoïdale, des aqueducs à éléments préfabriqués en terre cuite de même forme et de dimensions voisines, ou de section circulaire, présents dans l'est du département à Ginestas, à Mailhac (voir Harfouche et al., infra, p. 119-130). Ces conduites d'argile, dont on connaît un des lieux de fabrication, l'atelier de Sallèles-d'Aude (Laubenheimer $\ell$ al., 1990, p. 104 et fig. 70), pouvaient assurer un débit comparable, ou un peu inférieur ${ }^{86}$, pour des besoins de nature similaire. Ainsi deux modèles apparaissent-ils dans deux zones voisines du Languedoc : un modèle à canal maçonné, pour l'instant seul représenté dans l'ouest audois, et un modèle à canal en terre cuite plutôt présent dans la partie méditerranéenne de l'Aude et dans l'Hérault. Sans doute la production d'éléments préfabriqués marque-t-elle, dans ces régions plus sèches, le souci d'une meilleure efficacité et d'une rapidité accrue dans la réalisation des installations qui devaient y être plus nombreuses encore.

86. Dans le cas où l'élément en céramique constituc la totalité de la conduite, et non la partie inférieure d'une conduite de plus grande dimensions, comme au Traversant de Mailhac. Des tuyaux en terre cuite de section circulaire $\mathrm{et} \mathrm{de} \mathrm{plus} \mathrm{fort} \mathrm{diamètre} \mathrm{ont} \mathrm{été} \mathrm{découverts}$ dans l'atelier du Bourbou à Loupian (Hérault). 